\title{
VideoFlux. Una propuesta de investigación en torno al audiovisual experimental
}

\author{
Gabriel Villota Toyos ${ }^{1}$, Arturo fito Rodríguez ${ }^{1}$ \\ ${ }^{1}$ Grupo de Investigación Akmeka, UPV/EHU.
}

\begin{abstract}
The VIDEOFLUX project is proposed as an area of research and production related to experimental video and cinema as well as the recovery of videographic materials within the framework of the work carried out by the AKMEKA Research Group. In this sense, it crosses in a transversal way the research lines of this group, related to the "Audiovisual, technologies, networks: Experimentation and performativity and the one that attends to" Critical and political positions of art, culture and the media. The purpose of this communication is to publicize the group and its main objectives.
\end{abstract}

Keywords: Archive, obsolescence, technology, performativity

\section{Resumen}

El proyecto VIDEOFLUX se plantea como un área de investigación y producción relacionada con el audiovisual experimental, así como la recuperación de materiales videográficos en el marco del trabajo que lleva a cabo el Grupo de investigación AKMEKA. En este sentido atraviesa de manera transversal las líneas de investigación de este grupo, relacionadas con el "Audiovisual, tecnologías, redes: Experimentación y performatividad y la que atiende a "Posiciones críticas y políticas del arte, la cultura y los medios". Esta comunicación tiene como objeto dar a conocer el grupo y sus principales objetivos.

Palabras clave: Archivo, obsolescencia, tecnología, performatividad 


\section{El nacimiento de un nuevo proyecto}

El proyecto Videoflux que venimos a presentar aquí, nace como un área de investigación y producción relacionada con el audiovisual experimental, así como con la recuperación de materiales videográficos en el marco del trabajo que lleva a cabo el Grupo de investigación universitario Akmeka, de la Facultad de Bellas Artes de la Universidad del País Vasco / Euskal Herriko Unibertsitatea.

Pero antes de seguir nos preguntamos, ¿qué puede significar hoy en día investigar "en torno al audiovisual experimental", como se indica en el título? ¿y qué significa hacerlo "aquí y ahora", es decir, en el contexto artístico y académico del País Vasco y del conjunto de España en este, por otro lado, aciago 2021, y en el imprevisible mundo post-pandémico?

Responder a la primera pregunta significa, de entrada, plantearnos cuando menos otras dos nuevas cuestiones: como es, en primer lugar, pensar qué significa, en el actual momento de hegemonía de la imagen digital, de las plataformas y del consumo bajo demanda, el concepto mismo de "audiovisual experimental", en términos tanto ontológicos como materiales (o si se prefiere, en palabras de Donna Haraway, "semiótico-materiales"): no ya como sucediera en la Era de la MTV, la que constituyera el marco simbólico y mediático que marcó el conjunto de reflexiones anteriores, durante los años 80 y 90, sino en la nueva Era de YouTube, TikTok y Twitch, entre otras.

En segundo lugar, tendríamos que reflexionar también sobre una cuestión que tampoco es baladí, como es definir ese "hoy en día" que se menciona en la primera pregunta; y que creemos está marcado de manera rotunda por el hecho ineludible de una globalización que ya estaba presente o cuando menos intuida en las primeras teorías surgidas en torno al vídeo; las cuales habían nacido profundamente influidas ya por la visionaria idea de la "aldea global" macluhaniana, claramente identificable en aquellas pioneras emisiones a escala planetaria, realizadas vía satélite, como fuera "Wellcome Mr. Orwell"' (Nam June Paik, 1984).

Por otro lado, para responder a la segunda cuestión, relacionada con nuestro "aquí y ahora", inevitablemente habremos de dirigirnos a una cadena de textos y contextos que de forma interrumpida (o más bien a modo de Guadiana) han jalonado la historia del audiovisual experimental, tanto vasco como español, y que para nosotros de forma simbólica comenzaría con algunas de las primeras reflexiones autoconscientes surgidas a finales de los años ochenta, que se produjeron en el marco del Festival de Vídeo Bideoaldia en Tolosa y sus señeras publicaciones (González: 1989, Bonet: 1989, Palacio: 1989); que fue continuada después ya en los noventa por artículos aparecidos en 
revistas como Ars Video y publicaciones monográficas como "Plusvalías de la imagen" (Expósito y Villota: 1993), catálogos de muestras especialmente relevantes como "Señales de vídeo" (Bonet: 1995), y a finales de esa década, los dos volúmenes surgidos alrededor de los Encuentros de Vídeo de Pamplona (1996, 1998), entre muchos otros. Todo este recorrido será, ya en el nuevo siglo, minuciosamente recopilado y catalogado en contextos como los del macro-proyecto "Desacuerdos" (tanto en su fase de investigación y exposición, a través de proyectos como "Devenir Vídeo" [Villota, 2004] como en las sucesivas publicaciones vinculadas al mismo: destaquemos particularmente el trabajo desarrollado por Ignacio Estella en Desacuerdos no4, 2007), y también se verá continuado por otras iniciativas independientes como "Caras B del vídeo arte español" (Aramburu, T. Mori: 2011). En todo caso es palpable que hasta ese momento la inmensa mayoría de reflexiones producidas en el contexto español en torno al estado del "audiovisual experimental" en los últimos tiempos (aunque como enseguida veremos, no siempre fue así) se venían produciendo FUERA del entorno académico, salvo algunas débiles iniciativas tentativas, aquí y allá.

En ese sentido, supone para nosotros un cambio de perspectiva de la mayor relevancia la aparición a partir de 2014 de un proyecto como ARES, cuando por fin podemos asistir a la puesta en escena del compromiso académico universitario con todo el legado de producción y reflexión del audiovisual experimental español. Tanto el repositorio de trabajos online, como las publicaciones que se han ido editando, así como este mismo congreso, son claras muestras de un camino a seguir, y al que gustosamente queremos sumarnos.

\section{Todos tenemos un pasado (primera digresión)}

Pero responder a las anteriores preguntas desde el "aquí y ahora" también puede ser algo que explique nuestra irrupción como colectivo investigador VideoFlux desde el contexto universitario vasco en estos precisos momentos: y es que, obviamente, nuestra propuesta no surge ex nihilo.

En primer lugar, habría que remitirse a una pionera generación de profesores del entonces Departamento de Audiovisuales de la Facultad de Bellas Artes ${ }^{1}$, en la propia UPV/EHU, quienes desarrollaron una serie de tesis doctorales a comienzos de los noventa que han sido referencia para multitud de investigadores posteriores, tanto dentro como fuera de la Academia, y tanto dentro como fuera del propio País Vasco: me refiero a los textos publicados por Josu Rekalde (1988), Ander González Antona

\footnotetext{
1 En tiempos recientes este ha desaparecido como tal, para quedar subsumido en un departamento más amplio de Escultura y Arte y Tecnología.
} 
(1995), Juan Crego (1995), entre otros. Posteriormente, y a través de sucesivos grupos y proyectos de investigación, se ha ido ampliando ese campo de trabajo que ellos iniciaron, pero al mismo tiempo, bien por una especialización sobre algunos aspectos concretos (performance, sonido, interactividad, etc.), bien por una deriva natural hacia el agotamiento, llevábamos ya demasiado tiempo, casi podríamos decir un par de décadas desde que comenzara el nuevo siglo, con una creciente pérdida de foco alrededor de aquello que ahora volvemos a definir con el nombre y el adjetivo que creemos mejor sigue respondiendo al objeto de nuestras cuitas: el Audiovisual Experimental, en toda la extensión del término.

Proyectos de investigación como el que nos proponemos iniciar se entroncan, pues, en una cierta tradición de reflexión en torno a estas materias que se ha ido dando en nuestra propia universidad, pero que sin embargo consideramos que en tiempos recientes había ido quedando relegada, y necesitada por tanto de coger nuevos vuelos (además de producirse en el marco de lo que podríamos llamar un natural "relevo generacional", consecuencia de las jubilaciones).

Por otro lado, no es ningún secreto que el País Vasco ha sido un entorno especialmente fecundo en la escena española del vídeo experimental desde los mismos años ochenta, habiendo dado lugar a multitud de encuentros y festivales (Festival de Vídeo de Donostia, Bideoaldia de Tolosa, Festival de Vídeo Musical de Vitoria, etc.), alguno de los centros de producción y consulta más relevantes (talleres y Mediateca de Arteleku, etc.), distribuidoras (Ars Video), programaciones permanentes (Off Video, Sala Rekalde) publicaciones, y sobre todo, mucha producción por parte de los artistas, no pocas veces sostenida con una política pública de becas y subvenciones por parte de las diversas instituciones locales.

Como se viene investigando a través de las aportaciones de otros miembros de nuestro colectivo VideoFlux, la deriva de la colección de cintas del centro artístico Arteleku (perteneciente a la Diputación Foral de Gipuzkoa) ha devenido una cuestión particularmente paradigmática para nosotros, tanto en términos de investigación sobre los procesos de obsolescencia de los soportes, como de las propias derivas de la responsabilidad institucional (véase en particular la ponencia aquí presentada del investigador Pablo Maravi Martínez).

Ese será por tanto el cruce de caminos donde nos situaremos, animados por la proyección que propuestas como la surgida alrededor de ARES está teniendo en los años más recientes. 


\section{Situación actual: el "aquí y ahora" (segunda digresión)}

Llegados a este cruce de caminos, asumir que el panorama ha cambiado sustancialmente respecto a aquel paisaje del que proveníamos, no significa que haya dejado de ser pertinente la labor investigadora, sino que a partir de ahora habrán de tomarse en cuenta nuevos parámetros desde los que desarrollarla. $Y$ es que, ciertamente, son muchas las cosas que han cambiado respecto a aquella década de los años noventa en la que se configuró el principal esfuerzo teórico y especulativo en torno al vídeo. Estas serían las principales:

1. la Red de Festivales de Vídeo, con sus características itinerancias, y otras sinergias, prácticamente ha desaparecido como tal, aunque en su lugar sobrevivan ciertas experiencias similares, generalmente mucho más aisladas, especializadas y/o comerciales.

2. los Centros de Documentación principales de aquel periodo, y esto es más sorprendente, están inexplicablemente desaparecidos también: a la muerte en 2013 del centro de consulta, catalogación y documentación de la Mediateca de la Caixa ${ }^{2}$, pionero entre otras cosas de la tarea de digitalización de todo su material y de la puesta de consulta online (hoy igualmente desaparecida), se sumará la de la colección de la mediateca de Arteleku, heredera del legado de Bosgarren Kolektiboa (Bideoaldia de Tolosa) y en general testigo de todo el "estado de las cosas" del Vídeo de los ochenta y noventa, como ha estudiado Pablo Maravi: muerte tanto física como digital, en tanto que a la inundación provocada por el desbordamiento del Urumea, que asoló el centro en 2011 , destrozando una cantidad importante de las cintas, se sumó la desactivación y el descuido técnico respecto a la colección digital, llevada a acabo en los años previos a su errático desplazamiento desde el Convento Santa Teresa en Donostia (durante el año 2015, en que el hasta entonces diverso proyecto de Arteleku quedó reducido al monacal y monocorde espacio de "Kalostra" ${ }^{3}$ ), para pasar luego a un desubicado emplazamiento en Tabakalera, sin que aún a fecha de hoy siga sin saberse bien de quién es exactamente responsabilidad: si de Ubik -mediateca del centro-, de la Escuela de Cine Elías Querejeta, del centro Koldo Mitxelena, de Filmoteca Vasca, o simplemente de Tabakalera como tal).

3. La desaparición también de las publicaciones especializadas, cuyos objetos de estudio han ido quedando paulatinamente subsumidos en publicaciones más

\footnotetext{
${ }^{2}$ Merced al trabajo ejemplar desarrollado durante muchos años por Toni Mercader y Carme Garrido.

${ }^{3}$ https://www.noticiasdegipuzkoa.eus/cultura/2015/01/10/kalostra-sustituye-arteleku-escuelaexperimental/362409.html
} 
generalistas, bien sea en torno al audiovisual entendido de forma genérica, bien sea en torno a las nuevas prácticas artísticas y documentales contemporáneas, especialmente aquellas vinculadas al mundo digital.

4. La presencia en los Centros de Arte y Museos, que desde finales de los noventa ha ido adquiriendo una relevancia exponencial, tanto respecto a la historización, catalogación y recuperación de aquellos artistas y experiencias "históricas", como en relación a la incorporación de las nuevas generaciones de artistas que de forma creciente vienen usando formas de expresión vinculadas al "audiovisual": hasta el punto de que podríamos hablar de un proceso hegemónico de “audiovisualización" del sistema del Arte Contemporáneo (Villota, 2003).

5. La propia producción de obras de carácter "audiovisual experimental", ya no queda circunscrita a un territorio específico dentro del mundo del arte (el del "videoarte", o el de las llamadas, de forma más genérica, "nuevas tecnologías"), sino que se ha visto ampliamente extendida a diversos ámbitos del trabajo "creativo" en soporte vídeo: así, hoy en día podemos encontrar claras trazas de expresión experimental en productos audiovisuales pertenecientes a ámbitos de creación claramente delimitados/escindidos entre si, como es el del Vídeo Musical, los Videojuegos, la Animación, los trabajos de corte Interactivo, el Documental y sus nuevas formas, las producciones creadas para la Red, etc., más obviamente aquellos trabajos que se crean y consumen dentro de la lógica comercial del mundo del Arte Contemporáneo (centros de arte, galerías, ferias), como ya hemos dicho.

Creo por tanto que estas serán las nuevas claves que nos permitan entender de qué mimbres está constituido el "aquí y ahora" del audiovisual experimental sobre el que debemos investigar: tarea por lo demás ineludible, toda vez que dichos procesos de "audiovisualización" y expansión del "propósito experimental" a campos limítrofes están generando un nuevo y rico hábitat sobre el que conviene urgentemente reflexionar.

A todo esto, habríamos de añadir como elemento característico del momento presente algunas de las paradojas que actualmente se dan en la llamada "Iconosfera Contemporánea" (Gubern, 1987) o la "pantalla global" (Lipovetsky, 2009), caracterizada fundamentalmente por el hecho ineludible de la hegemonía de la imagen digital.

Es sumamente curioso advertir cómo, por ejemplo, tras décadas en las que la imagen vídeo (soportada en cinta magnética) nunca dejó de sufrir las polémicas con la imagen cine (registrada sobre película de celuloide o soporte fílmico), aguantando estoicamente la mirada de superioridad de la segunda desde su pretendido pedigrí y mayor definición de la imagen, así como por su carácter más "auténtico" frente al 
supuestamente carácter artificial del vídeo (la reproducción de base binaria del vídeo, como flujo electrónico fruto de la alternancia de dos campos visuales, frente a la mayor riqueza de la imagen-luz analógica, huella pura), hemos llegado a un momento en que, siendo TODAS las imágenes que vemos en la esfera pública de base digital, sigue valorándose la imitación de aquellos estilemas y signos que nos remiten simbólicamente al "fallo" analógico presente en la textura/grano de la imagen (super8, $16 \mathrm{~mm})$, los "rayones" del celuloide, los "velados" accidentales de los procesos de revelado, etc.: todo ello convenientemente ofrecido en los menús de efectos de programas de edición y posproducción de imagen y sonido como Photoshop, Premiere etc., o incluso en los de aplicaciones como Instagram.

Definir en este sentido qué es hoy en día "vídeo", si apelamos por ejemplo a la base material del soporte de registro, resulta relativamente simple, dado que la respuesta es: hoy todo es vídeo (Villota, 2017). Vídeos son, por extensión, los que emite Youtube como repositorio infinito, o aún mucho más los miles de millones que se graban a diario con los teléfonos móviles y se difunden sin cesar por redes como Instagram, Twitter, Whatsap, Facebook o TikTok. Pero también los que graban los reporteros de televisión, las cámaras de vigilancia, los documentalistas, y los artistas. Incluso, las películas de los cineastas, tanto las underground como las de Hollywood. Nada audiovisual existe ya fuera del vídeo.

\section{La propuesta concreta}

Volviendo entonces al motivo de nuestra presentación veamos pues cuáles son, en el contexto descrito, los objetivos precisos de este proyecto, y cómo el equipo de VideoFlux los está comenzando a desarrollar, atendiendo a una metodología específica que queremos presentar aquí. Así fueron enunciados en la primera presentación pública del mismo:

- Dar a conocer un prolífico territorio creativo de nuestro contexto inmediato a través del cuidado y la preservación de los materiales de la Facultad, así como de otros centros. Revisar la idea de archivo audiovisual.

- Investigar y plantear una publicación que se ocupe del ámbito audiovisual y tecnológico, de sus circunstancias actuales y de sus retos, mediante una serie de textos que están ya en preparación. Posible publicación académica.

- Generar un material que pueda servir como recurso docente y de investigación, que pueda ser incluido en los programas educativos y académicos. Necesidad que percibimos en la oferta docente de la Facultad de Bellas artes ante la ausencia de asignaturas relacionadas con el audiovisual experimental, especialmente en cuarto curso. Posible publicación materiales docentes. 
- Producir nuevos materiales audiovisuales a través del reciclaje y la interpretación del archivo audiovisual que sirve de núcleo a esta investigación para interpelar a un público amplio que incluya particulares, profesores, estudiantes, investigadores, artistas, comisarios... Posible producción audiovisual / Jornadas temáticas / Evento.

Como se ve en esta enumeración de objetivos se atiende por tanto a un diríamos triple frente de batalla, en el que de un lado están presentes los trabajos de recuperación, catalogación y archivo (a partir de los dos casos consignados: Arteleku, y la videoteca de la propia Facultad de Bellas Artes; pero yendo más allá en el intento de recuperar otras cintas y autores no presentes en esas colecciones):

El proyecto de investigación propone una mirada a la historia de creación audiovisual en el País Vasco a través de una metodología de trabajo alejada de la historiografía convencional, intentado evitar la visión única y los formatos convencionales. Se trata de asumir un proceso de trabajo colectivo de exploración, una metodología en la que se interpele directamente a los distintos protagonistas del objeto de estudio y en la que se valore sus experiencias y sus aportaciones en un momento histórico determinado. Se trata de catalogar todos estos datos para reinsertarlos en la escena actual y dotarlos de sentido en el contexto del audiovisual contemporáneo, pero también en el contexto general del arte y en el contexto cultural y social del País Vasco.

La impronta audiovisual que invade la actividad del arte contemporáneo y que ha propiciado el tránsito de la denominación de "Artes plásticas" a "Artes Visuales", hace preciso el volver sobre algunos episodios de la historia del arte en Euskal Herria, para poner en valor ciertos hitos, nombres, obras, movimientos, actuaciones y circunstancias de experimentación artística y tecnológica.

En segundo lugar, se trata también de abundar en el trabajo de análisis y reflexión crítica en torno al actual "estado de la cuestión" del vídeo, que pueda verse culminado tanto con publicaciones de tipo académico como con otras más divulgativas y/o enfocadas en la docencia.

$Y$, en tercer lugar, queremos destacar la importancia de considerar en este proyecto la práctica artística misma como actividad investigadora, lo cual en este caso, como explicamos más adelante, quedará planteado a partir del desarrollo de una metodología híbrida que supondría producir trabajos audiovisuales de tipo 
"desmontaje" ${ }^{4}$, a partir del reciclado y la apropiación de los mismos materiales estudiados en el archivo, o incluso contemplando la posibilidad de que estos sean llevados al terreno performativo en sesiones de "directo", que terminarían por producir una suerte de celebración del trabajo colectivo y de cuestionamiento de los formatos tradicionales de "exhibición":

El proyecto se articula principalmente a través de diversos ejes: Producción, Creación, Exhibición y Difusión. Cada uno de ellos reclama planteamientos independientes pero complementarios. La propuesta de investigación no solo se perfila como un estudio multidisciplinar, sino que se ve afectada por una transversalidad real que da cuenta de los escenarios creativos, de los parámetros sociológicos y de las políticas culturales en los que se inscribe.

Creemos en este sentido que una de las fortalezas de nuestra propuesta será este intento de interrelacionar la parte más académica / teórica, con una parte más práctica conformada tanto a través de la labora archivística como de la creadora: es para ello un antecedente importante el proyecto "Ikusmirak / Formas de ver" (Arturo fito Rodríguez, Gabriel Villota, 2013), en el que precisamente se definían las bases de este tipo de práctica investigadora híbrida. Decíamos allí:

El reto principal de esta propuesta estriba precisamente en el experimento metodológico, esto es, en la búsqueda de un método lo suficientemente coherente en su experimentación como para hilvanar propuestas de raíz y procedencia muy dispar. Se trataría de trabajar sin la presión de una perspectiva historicista que obture las posibilidades de una articulación subjetiva de las obras; se trataría de trabajar sin la intención de crear rígidas genealogías o ascendencias regias, pero sugiriendo a la vez parentescos o consanguinidades negadas o arrebatadas. Se trataría también de trabajar conociendo la complejidad que surge de la adecuación de los materiales audiovisuales a la sala de exposición y, por tanto, considerando metodológicamente y desde el primer momento que una propuesta como esta debe trasladar su característica experimental al display expositivo.

Esto en lo que respecta a la manera de abordar las nóminas de artistas y trabajos, y las correspondientes genealogías en este caso obliteradas. Pero en lo que se refiere a los modos de articular ese material, allí se presentaban también algunas propuestas que ahora pretendemos rescatar:

\footnotetext{
${ }^{4}$ Título de la muestra y publicación seminal dirigida por Eugeni Bonet a comienzos de los 90, como primera gran reflexión habida en nuestro país en torno al uso de la apropiación en el cine y vídeo experimental (Arteleku, IVAM, 1990).
} 
Un aspecto metodológico que nos hemos planteado también desde el primer momento es la posibilidad de sustentar una parte importante de la investigación en la toma de imagen, en la grabación en video de distintas entrevistas o conversaciones con autores, estudiosos o agentes vinculados al contexto de la muestra. Aparece también desde la puesta en marcha de este proyecto la idea de que esta suerte de "trabajo de campo" que son las grabaciones de video participen de la propia exposición, ya sea como documentación adicional o como itinerario. Los video-documentos resultantes, la propia investigación, se incorporaría así a la muestra como una hoja de ruta, indicando los caminos elegidos y su articulación. Se buscaría con ello ofrecer una coherencia entre el soporte de investigación, el soporte informativo o comunicativo de la exposición y las obras audiovisuales que se muestran. El objetivo no sería otro que dar sentido a las vías o los caminos elegidos mediante una sintaxis audiovisual, huyendo del didactismo, pero ofreciendo apoyos para su comprensión.

Como ya se planteaba allí, en el proyecto actual nos proponemos por tanto abordar un proceso experimental e investigador que parta de los materiales del archivo de la Facultad, y que continúe con la generación colectiva de un tejido audiovisual hecho de la reutilización y la remezcla, como nuevo modo de reactivar por un lado esos materiales diversos que quedaron en archivos más bien olvidados (más allá de su oportuna restauración y digitalización), y con el propósito de que a su vez puedan cobrar nuevos significados a través el montaje; sumando a ello fragmentos registrados de nuevo cuño, consistentes como decíamos en entrevistas, debates u otros.

Por otro lado, también creemos que la difusión de este material forma parte del proyecto y del proceso de investigación mismo, máxime habida cuenta del tipo de objeto de estudio del que venimos hablando: es por ello que estamos valorando igualmente ideas acerca de cómo llevar estos trabajos al espacio expositivo, por ejemplo, viendo qué tipos de dispositivos usar, y cómo combinarlos:

Sabemos que trabajamos con el espacio codificado del arte que es la sala de exposición, y que en esta ocasión trabajamos especialmente con el tiempo (de la obra, del visionado, del espectador, con "el tiempo de la exposición").

Sin duda el montaje de la exposición es una parte esencial y en cierto modo justificadora del proyecto de investigación. Aún siendo este un factor que se define en el proceso, si podemos decir que buscamos desde ahora una fórmula que permita el acceso a las obras en las mejores condiciones y que en la medida de lo posible venga a aportar algunas soluciones a ese "cine de exposición" del que habla Jean-Christophe Royoux. Por ello es nuestro propósito revisar las condiciones 
históricas que hacen hoy día posible la confluencia de ambos campos: cine y exposición. Se trata en todo caso de concebir la sintaxis de un dispositivo espacial que funciona por sí mismo y que se aventure como verdadera máquina de relación directa con la obra propiciando el redescubrimiento de artistas y de cineastas.

Unido a todo ello, nos gustaría también reivindicar la posibilidad de introducir el dispositivo cinematográfico del cine en la sala de exposición, precisamente para evidenciar la posibilidad de convivencia de los distintos medios. La sala de exposición de artes visuales es la que tiene que favorecer esta convivencia, en la sala se haría realidad lo que el discurso teórico argumenta fuera de ella. La sala de exposición recoge por fin físicamente una sensibilidad manifestada también en la práctica contemporánea.

Igualmente queremos valorar la posibilidad de disfrutar de las distintas texturas audiovisuales que proporcionan los distintos medios, ya que el espacio de la sala de exposiciones es el lugar en donde poder dar cuenta de esta diversidad y de esta riqueza formal, sensible. La propuesta quiere de algún modo dar cuenta de toda esa riqueza, de sus transformaciones y de sus hibridaciones.

A fin de no ser crípticos con nuestra propuesta, pero para evitar caer en la habitual tendencia a inundar la sala con textos informativos que anulen la lectura propia del visitante, proponemos la idea de generar una hoja de ruta audiovisual (pequeños monitores) que sustituyan o complementen a las informaciones de vinilo en la pared. Lo mismo sucedería con las piezas audiovisuales de cierta duración: está en nuestra mano la información de estas duraciones al público, dando la posibilidad de articular en algún caso "ciclos" o "pases" de ciertas obras y a ciertas horas dentro de la propia sala para facilitar el que cada visitante pueda programar su visita Un espacio mixto que haga posible compatibilizar todos los formatos de exhibición. Se trataría, en fin, de dar soluciones a todas estas cuestiones a partir del material seleccionado o generado. De fondo a todo este campo de operaciones se encuentra también el análisis de los regímenes de visibilidad que operan sobre este tipo de obras en el contexto del museo y de la galería de arte.

Y finalmente también estamos estudiando la posibilidad de que, más allá de una mera exhibición "pasiva", la muestra del proyecto pudiera también desarrollarse a través de un formato más performativo, dentro del modelo ya desarrollado en propuestas anteriores de las "batallas de pantallas": 
En más de una ocasión hemos sentido que los formatos y los modos de presentación podrían adoptar formas y fórmulas más eficaces, capaces de ofrecer nuevas posibilidades tanto creativas como comunicativas, en el intento de provocar conexiones con otros ámbitos de conocimiento o de generar nuevos debates.

Se trata también de pensar una vez más sobre la idea de audiencia, sobre la pertinencia de "crear públicos", de robarlos o de hacerlos desistir, todo ello con el panorama explosionado de la red como elemento fundamental de relación del audiovisual.

La propuesta "Batalla de pantallas" busca un tipo de presentación abierta y dinámica, con capacidad para la deriva, el cruce de temas y la participación activa de los asistentes. En base a una serie de "tags" que se plantean como punto de partida (que se publicitarán en la comunicación del evento), y que en este caso toman como referencia los ejes de nuestro proyecto, los conductores trabajan por separado recopilando citas visuales, textos y comentarios.

El trabajo de investigación previo está enfocado a recopilar una serie de documentos audiovisuales, sitos web, artículos, citas textuales y visuales que vengan a ilustrar, desmontar o implementar dichos puntos de partida.

La articulación y el cruce de estas referencias en vivo y en directo mediante tres proyecciones simultáneas (un ordenador y un proyector por cada conductor), se plantea en base a un diálogo abierto y mínimamente estructurado, ofreciendo lecturas cruzadas, improvisaciones, nuevas búsquedas en internet, comentarios de los asistentes...

Esta es, por tanto, en definitiva, nuestra propuesta para retomar, en nuestro contexto particular, la que con cierta urgencia consideramos necesaria investigación en torno al audiovisual experimental, en el momento presente. Iniciativas como ARES son sin duda un acicate importante para que este equipo que hemos montado nos hayamos animado a ello, con la ilusión y en la confianza de que encuentros como este generen las sinergias necesarias que nos permitan poner de nuevo la investigación "en torno al vídeo" en el centro del debate artístico, tal y como creemos la coyuntura actual del arte y la cultura lo necesita. 


\section{Referencias}

ARAMBURU, Nekane, T. MORI, Carlos (EDS.) (2011): Caras B del vídeo arte español. Madrid: Agencia Española de Cooperación Internacional para el Desarrollo.

BONET, Eugeni (1989): “Madrid 84-86; Vídeo español, el mejor vídeo de España”, en Catálogo del Bideoaldia'89. Manual de Instrucciones II. Tolosa: Bosgarren Kolektiboa.

BONET, Eugeni (ED.) (1995): Señales de vídeo. MNCARS, Madrid.

BONET, Eugeni (2010): “Alter-Video”, en En torno al vídeo. Gustavo Gili, Barcelona 1980; Fundación Rodríguez, UPV/EHU, Bilbao.

CREGO, Juan (1995): La institucionalización del videoarte en España : influencia de las instituciones en un arte en formación. Servicio Editorial UPV/EHU, Bilbao.

ESTELLA, Ignacio (2007): “Cuando las actitudes devienen norma. Institucionalizaciones del vídeo en el Estado español”, en Desacuerdos № 4. Arteleku, Centro José Guerrero, MACBA, UNIA (Granada).

EXPÓSITO, Marcelo y VILLOTA, Gabriel (EDS.) (1993): Plusvalías de la imagen. Anotaciones (locales) para una crítica de los usos (y abusos) de la imagen. Bilbao: Sala de Exposiciones REKALDE, S.L.

GONZÁLEZ-ANTONA, Ander (1995): El video en el País Vasco (1972-1992): reflexiones en torno a una práctica artística del video. Servicio Editorial UPV/EHU, Bilbao.

GONZÁLEZ, Xabier (1988): "Vídeo vasco: de inexistente a desconocido", en Catálogo I Festival de Vídeo de Canarias. Gran Canaria: Cabildo Insular de Gran Canaria, Comisión de Cultura.

GUBERN, Román (1987): La mirada opulenta. Barcelona, Gustavo Gili.

LIPOVETSKY, Gilles, SERROY, Jean: La pantalla global: Cultura mediática y cine en la era hipermoderna (Barcelona, Anagrama, 2009).

MARTíNEZ COLLADO, Ana, y PANEA, José Luis (EDS.) (2017): Secuencias de la experiencia, estadios de lo visible. Aproximaciones al videoarte español. Universidad de Castilla la Mancha, Brumaria, Madrid.

REKALDE, Josu (1988): Una experimentación en el campo del video-arte fundamentada en la noción de transformación temporal. Servicio Editorial UPV/EHU, Bilbao.

RODRÍGUEZ BORNAETXEA, Arturo fito, VILLOTA TOYOS, Gabriel (2018): “Notas para un paseo por las prácticas audiovisuales experimentales en el País Vasco 1968-2016", en Video-Arte. Recorridos por la creación videográfica en Euskal Herria. BilbaoArte, UPV/EHU, Bilbao.

VVAA (1996): Encuentros de vídeo de Pamplona. Pamplona.

VVAA (1998): Encuentro de vídeo y Altermedia de Pamplona (Pamplona, 
VILLOTA TOYOS, Gabriel (2003): “Espectáculo y devenir audiovisual en la escena artística contemporánea", Revista de Occidente n. 261, Madrid.

VILLOTA TOYOS, Gabriel (2017): “De la identidad al flujo. El vídeo como híbrido de tecnología y cuerpo", en Secuencias de la experiencia, estadios de lo visible. Aproximaciones al videoarte español. Universidad de Castilla la Mancha, Brumaria, Madrid.

VILLOTA TOYOS, Gabriel (2007): Devenir vídeo (adiós a todo eso) (180', 2005, dvd), en revista Desacuerdos no 4 MACBA, UNIA, Arteleku, Centro José Guerrero, Granada. 